\title{
Opposition effect on comet 67P/Churyumov-Gerasimenko using Rosetta-OSIRIS images
}

\author{
N. Masoumzadeh ${ }^{1, \star}$, N. Oklay ${ }^{1}$, L. Kolokolova ${ }^{11}$, H. Sierks ${ }^{1}$, S. Fornasier ${ }^{13}$, M. A. Barucci ${ }^{13}$, J.-B. Vincent ${ }^{1}$, \\ C. Tubiana ${ }^{1}$, C. Güttler ${ }^{1}$, F. Preusker ${ }^{22}$, F. Scholten ${ }^{22}$, S. Mottola $^{22}$, P. H. Hasselmann ${ }^{13}$, C. Feller ${ }^{13}$, C. Barbieri ${ }^{4}$, \\ P. L. Lamy ${ }^{5}$, R. Rodrigo ${ }^{6,7}$, D. Koschny ${ }^{8}$, H. Rickman ${ }^{9,10}$, M. F. A'Hearn ${ }^{11,12,1}$, J.-L. Bertaux ${ }^{14}$, I. Bertini ${ }^{2}$, \\ G. Cremonese ${ }^{15}$, V. Da Deppo ${ }^{16}$, B. J. R. Davidsson ${ }^{9}$, S. Debei ${ }^{17}$, M. De Cecco ${ }^{18}$, M. Fulle ${ }^{20}$, A. Gicquel ${ }^{1}$, \\ O. Groussin ${ }^{5}$, P. J. Gutiérrez ${ }^{21}$, I. Hall ${ }^{1}$, M. Hofmann ${ }^{1}$, S. F. Hviid ${ }^{22}$, W.-H. Ip ${ }^{23}$, L. Jorda ${ }^{5}$, H. U. Keller ${ }^{3}$, \\ J. Knollenberg ${ }^{22}$, G. Kovacs ${ }^{1}$, J.-R. Kramm ${ }^{1}$, E. Kührt ${ }^{22}$, M. Küppers ${ }^{24}$, L. M. Lara ${ }^{21}$, M. Lazzarin ${ }^{4}$, \\ J. J. Lopez Moreno ${ }^{21}$, F. Marzari ${ }^{4}$, G. Naletto ${ }^{25,2,16}$, X. Shi ${ }^{1}$, and N. Thomas ${ }^{19}$
}

(Affiliations can be found after the references)

Received 16 September 2016 / Accepted 11 November 2016

\begin{abstract}
Aims. We aim to explore the behavior of the opposition effect as an important tool in optical remote sensing on the nucleus of comet 67P/ Churyumov-Gerasimenko (67P), using Rosetta-OSIRIS images acquired in different filters during the approach phase, July-August 2014 and the close flyby images on 14 of February 2015 , which contain the spacecraft shadow.

Methods. We based our investigation on the global and local brightness from the surface of 67P with respect to the phase angle, also known as phase curve. The local phase curve corresponds to a region that is located at the Imhotep-Ash boundary of 67P. Assuming that the region at the Imhotep-Ash boundary and the entire nucleus have similar albedo, we combined the global and local phase curves to study the opposition-surge morphology and constrain the structure and properties of 67P. The model parameters were furthermore compared with other bodies in the solar system and existing laboratory study.

Results. We found that the morphological parameters of the opposition surge decrease monotonically with wavelength, whereas in the case of coherent backscattering this behavior should be the reverse. The results from comparative analysis place 67P in the same category as the two Mars satellites, Phobos and Deimos, which are notably different from all airless bodies in the solar system. The similarity between the surface phase function of 67P and a carbon soot sample at extremely small angles is identified, introducing regolith at the boundary of the Imhotep-Ash region of $67 \mathrm{P}$ as a very dark and fluffy layer.
\end{abstract}

Key words. planets and satellites: surfaces - techniques: photometric - comets: individual: 67P/Churyumov-Gerasimenko

\section{Introduction}

Launched in 2004, the Rosetta spacecraft woke up on 20 January 2014 after ten years of cruising and 30 months of deep space hibernation. In July-August 2014, Rosetta reached the comet 67P/Churyumov-Gerasimenko (67P). Since then, the spacecraft has escorted the comet and observed it by a number of remote-sensing instruments (Schulz et al. 2009). OSIRIS, the Optical, Spectroscopic, and Infrared Remote Imaging System (Keller et al. 2007), was the scientific imaging system onboard Rosetta. It contained two cameras: the Narrow Angle Camera (NAC), and the Wide Angle Camera (WAC), which covered the wavelength range of $250 \mathrm{~nm}$ to $1000 \mathrm{~nm}$ with total of 26 filters. NAC and WAC were designed as a complementary pair to study aspects of the nucleus surface, such as its morphology (Thomas et al. 2015; El-Maarry et al. 2015) or its spectrophotometric properties (Fornasier et al. 2015), and to investigate the dynamics of the sublimation processes.

During the close flyby ( $\sim 6 \mathrm{~km}$ away from the comet surface) on 14 February 2015, zero-phase-angle observations were performed in combination of various filters. The images cover

\footnotetext{
* Corresponding author: N. Masoumzadeh, e-mail: masoumzadeh@mps.mpg.de
}

an area in the Imhotep-Ash boundary region (El-Maarry et al. 2015).

The close-flyby images of OSIRIS taken at very small phase angles help us to extend our understanding of the sharp increase in the brightness of airless bodies when the angle between incident light and the observer direction (phase angle $\alpha$ ) reaches zero. This phenomenon is called opposition effect (OE); it is of special interest in photometric studies and is a major remotesensing tool for understanding the nature of bidirectional reflectance from planetary regoliths.

The OE can be characterized by two parameters coming from the optical phase curve (dependence of brightness on phase angle) of a body. The $\mathrm{OE}$ parameters are the amplitude (also specified as the enhancement factor, $\zeta$ ) and the angular width estimated as the half-width at half-maximum, HWHM. The effect has been observed on various planetary surfaces (Belskaya \& Shevchenko 2000; Rosenbush et al. 2002; Shevchenko et al. 2008; Déau et al. 2009) and planetary regolith analogs in the laboratory (Shkuratov et al. 2002; Psarev et al. 2007; Déau et al. 2013; Jost et al. 2016).

From the theoretical modeling perspective, two mechanisms are proposed to explain the $\mathrm{OE}$, shadow hiding $(\mathrm{SH})$ and coherent backscattering (CB). The first is related to the amount of shadow that grains cast on each other (self-shadowing). The 


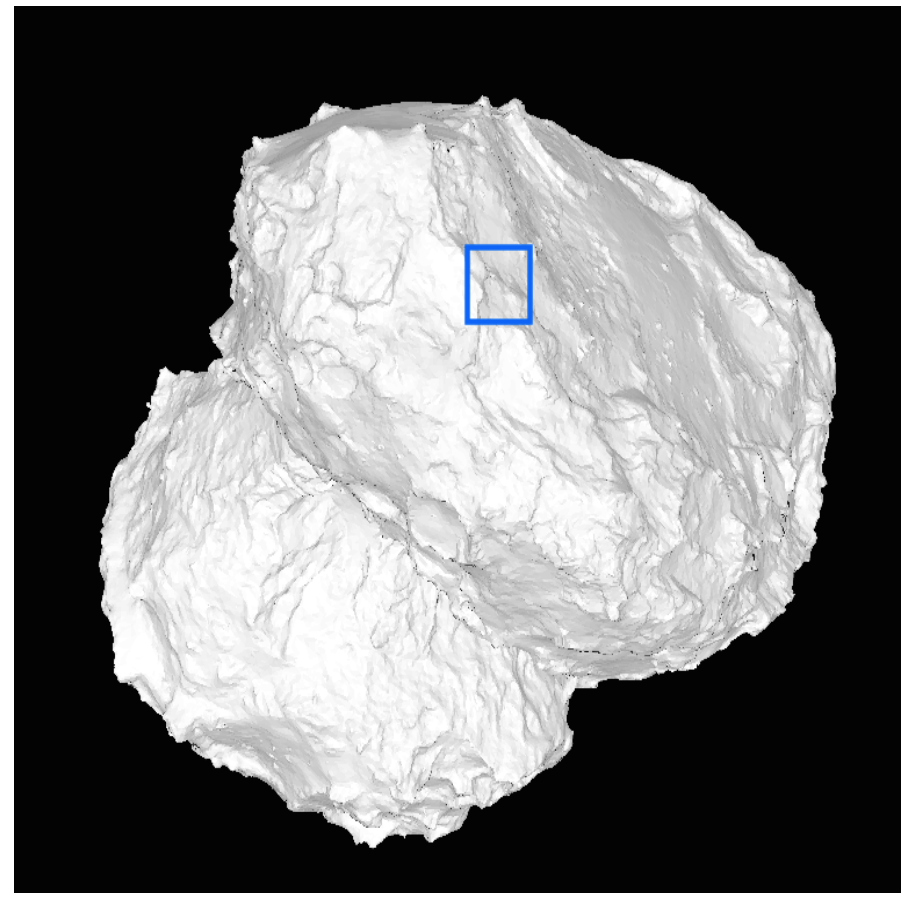

Fig. 1. Shape model of 67P outlined in blue, displaying the area imaged in the field of view of the OSIRIS NAC on 14 February 2015.

shadow cast by the regolith grains is hidden to the observer when $\alpha=0^{\circ}$ (Hapke 1986; Shkuratov 1994; Penttilä 2013). The second mechanism originates from constructive interference between the partial electromagnetic waves that travel in the medium in opposite directions and experience multiple scattering within the same path between particles. These waves leave the medium in phase and therefore provide the conditions for the constructive interference.

Recently developed theoretical models have gained some success in simulations of light scattering by particulate media (Mishchenko 1992; Muinonen et al. 2012; Mackowski \& Mishchenko 1996, 2011) to fully explain the observed OE. However, to simulate realistic media, these models require enormous computer resources, which are often unavailable to researchers, and deal with a limited range of parameters.

The goal of this paper is to study the opposition effect on the surface of comet 67P and the physical mechanisms behind this phenomenon. Accordingly, we constructed global and local surface phase functions of comet 67P using OSIRIS data that are described in Sect. 2. In Sect. 3, we analyze and illustrate the phase curves of comet 67P in different wavelengths for both the entire disk of the object and a small area on its surface. The results of the opposition-effect behavior of 67P are discussed in Sect. 5, which leads us to conclude on our main findings in Sect. 6.

\section{Observational data}

We used the OSIRIS NAC images taken in various filter combinations from 25 July to 6 August as well as three images taken during the close flyby on 14 of February 2015 to investigate the phase curves given in Sect. 3.1. The characteristics of images used in this study are given in Table A.1.

The July 2014 images were photometrically corrected using the Lommer-Seeliger (LS) disk function (Li et al. 2015), and geometrical angles were calculated from the global 3D shape model constructed by stereo-photoclinometric techniques (Jorda et al. 2016). The photometrically corrected images from August 2014 are taken from Fornasier et al. (2015).

In addition, the three flyby images (last row in Table A.1), which included the shadow of the spacecraft and, thus, were acquired at very small phase angles (less than $2^{\circ}$ ) were used. These three images, taken in F84 (480.7 nm), F82 (649.2 nm), and F88 (743.7 nm), were coregistered and photometrically corrected with the Lommel-Seeliger disk law using the USGS ISIS3 software (Integrated Software for Imagers and Spectrometers ${ }^{1}$, Anderson et al. 2004).

During the photometric correction, the angles were calculated using a 3D-shape model constructed by stereophotogrammetric methods (Preusker et al 2015) and SPICE kernels ${ }^{2}$. The details of image registration and photometric correction steps are described in Oklay et al. (2016).

All OSIRIS NAC images used in this study are expressed in the radiance factor, hereafter denoted as $I / F$. The radiance factor is defined as the ratio between the radiance of an illuminated surface and the radiance from a normally illuminated Lambert surface at the same observer-object separation.

The calibration of OSIRIS images is discussed in detail by Tubiana et al. (2015). The uncertainty of the absolute calibration is reported to be $1 \%-2 \%$ for NAC filters in the visible range.

\section{Analysis techniques and results}

To better understand how the surface scatters the sunlight, we need to cover a range of phase angles as broad as possible. For this, we supplemented the local phase curve, which covers very small phase angles, with the global phase curve with the larger phase angles. Then, we applied the approximation function used by Rosenbush et al. (2002) on the combined phase curve of 67P.

\subsection{Local phase curve}

Of the close-flyby OSIRIS images that have been acquired at small phase angles on 14 February 2015, the images including the spacecraft shadow (zero-phase-angle point) were used to extract the local phase curves. The top panel of Fig. 2 demonstrates the RGB image that captured the spacecraft shadow on the surface of the nucleus. Images taken in F88 (740 nm), F82 (649 nm), and F84 (480 nm) were coded to the RGB channels of the image. The Rosetta shadows are the colored patches at the bottom of the image.

The phase angle distribution map was computed for each facet using the shape model (SHAP4s with 1M facets Preusker et al. 2015) associated with SPICE kernels (Acton 1996) for Rosetta with the MATLAB software. The 2D phase angle map was superimposed on the multispectral image as a reference. The change in color indicates the phase angle variation with steps of $0.1^{\circ}$. The locations of regions of interest (ROIs) were chosen to collect the averaged $I / F$ corresponding to the different phase angles (see bottom panel of Fig. 2). The local phase curves in three wavelengths, spanning from $0.2^{\circ}$ to $2.0^{\circ}$, are displayed in Fig. 3. The behavior of the local phase curves can be described by a log-linear function approximation (Lamy et al. 2004),

$\log _{10} I / F(\alpha)=\log _{10} I / F_{0}+0.4 \alpha \beta$

\footnotetext{
http://isis.astrogeology.usgs.gov/index.html

2 http://www. cosmos.esa.int/web/spice/

spice-for-rosetta
} 
N. Masoumzadeh et al.: Opposition effect on comet 67P/Churyumov-Gerasimenko using Rosetta-OSIRIS images

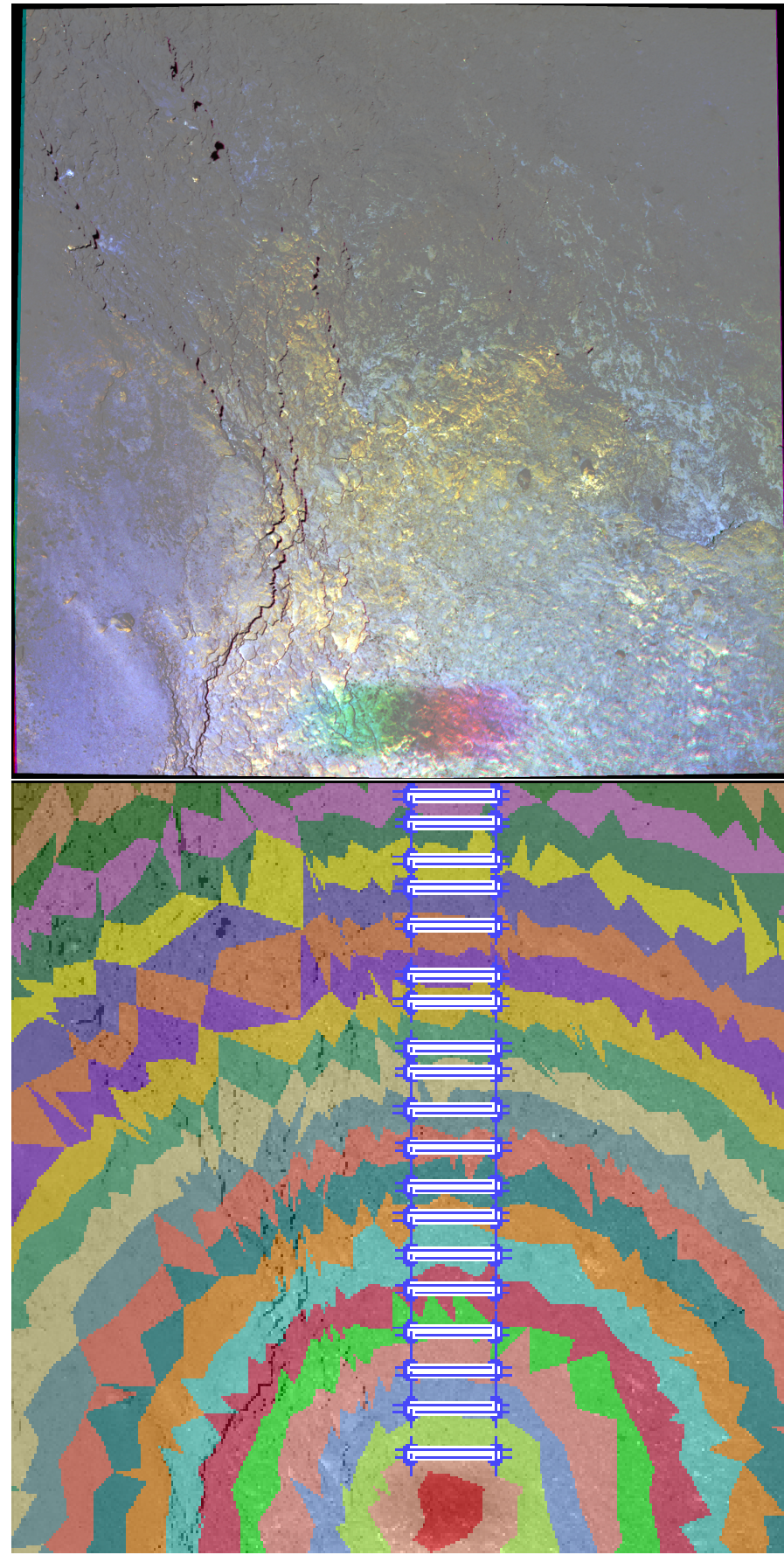

Fig. 2. Top panel: RGB image of the Imhotep-Ash boundary on the large lobe of 67P using OSIRIS NAC close-flyby images acquired on 14 February 2015 at UTC time of 12.39. The RGB images are associated with F88 $(740 \mathrm{~nm})$, F82 (649 nm), and F84 (480 nm). The colored patches are the spacecraft shadow. Bottom panel: phase angle distribution calculated for each facet using the DLR SPG model SHAP4s (1M facets) and overlay on the multispectral image. The colors are assigned to a phase angle step of $0.1^{\circ}$. The ROIs are outlined with blue boxes and contain 4400 pixels.

where $\beta$ is a phase coefficient in magnitude per degree (mag/deg), and $I / F_{0}$ gives the brightness at opposition $\left(\alpha=0^{\circ}\right)$.

The resulting phase coefficients are $0.086 \pm 0.025 \mathrm{mag} / \mathrm{deg}$ in the F84 $(480 \mathrm{~nm}), 0.0915 \pm 0.022 \mathrm{mag} / \mathrm{deg}$ in F82 $(649 \mathrm{~nm})$, and $0.0941 \pm 0.029$ in F88 $(740 \mathrm{~nm})$, respectively. The difference between the phase coefficients demonstrates a decrease in $\beta$ with

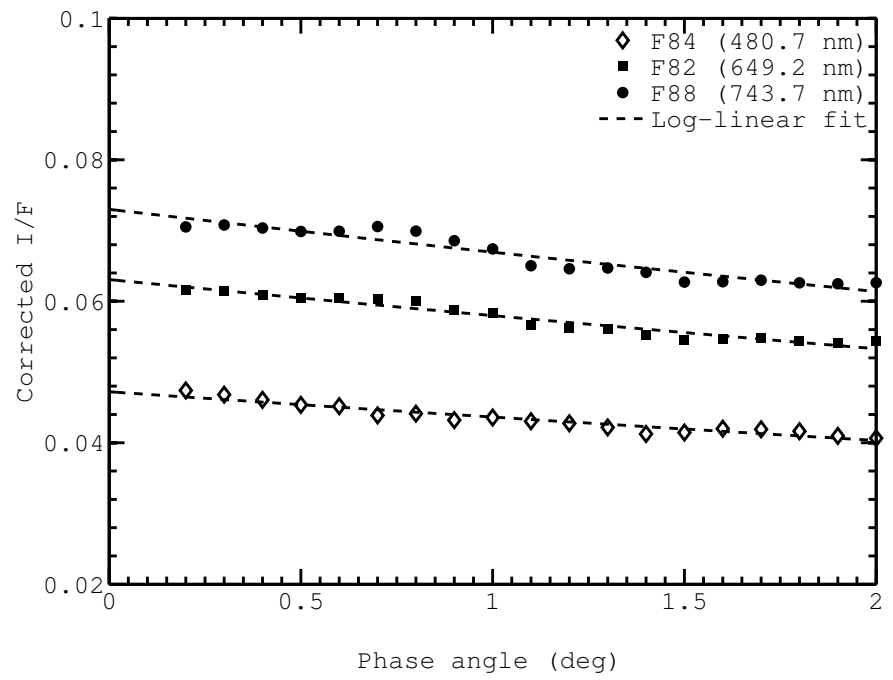

Fig. 3. Resulting local phase curves from the surface of $67 \mathrm{P}$ using OSIRIS NAC close-flyby images in three filters.

wavelength for the surface phase function of $67 \mathrm{P}$ at very small phase angles.

Because it was taken inside the opposition-surge part of the phase curve, the $\beta=0.0915 \pm 0.022 \mathrm{mag} / \mathrm{deg}$ in F82 $(649 \mathrm{~nm})$ from local phase curve in the range of $0.2^{\circ}$ to $2.0^{\circ}$ is steeper than those recorded for larger phase angles; (Tubiana et al. 2011) derived the linear phase coefficient of $0.061-0.076 \mathrm{mag} / \mathrm{deg}$ from the ground-based observations in the phase angle range of $0.5^{\circ}-$ $10^{\circ}$ and for $\alpha<15^{\circ}$, VIRTIS reported $\beta=0.082 \pm 0.016 \mathrm{mag} / \mathrm{deg}$ (Ciarniello et al. 2015) .

Since the effect of non-zero angular size of the Sun is a concern for objects in the inner and outer solar system, we computed the angular radius of the Sun for 67P using the following expression (Déau 2012 and references therein):

$\alpha_{\odot}=\arcsin \frac{R_{\odot}}{D_{67 \mathrm{P}-\odot}}$

where $R_{\odot}$ is the radius of the Sun $\left(6.955 \times 10^{5} \mathrm{~km}\right)$ and $D_{67 \mathrm{P}-\odot}$ is the distance between $67 \mathrm{P}$ and the Sun $\left(3.466 \times 10^{8} \mathrm{~km}\right)$.

The resulting value of the angular size of the solar disk as seen by $67 \mathrm{P}$ during the observation, $0.12^{\circ}$, indicates that the minimum phase angle of $0.2^{\circ}$ of the local phase curve is not within the angular size of solar disk. Hence, the size of the Sun does not influence our local phase curve.

\subsection{Global phase curve}

We built the global surface phase function ( $\mathrm{Li}$ et al. 2015) for the whole cometary nucleus, hereafter referred to as global phase curve, to extend the local phase curve as a means to model the morphology of the phase curve (filled symbols in Fig. 4). To construct the global phase curve of 67P, we used OSIRIS images taken from 25 July to 6 August (Table A.1) in the phase angle range of $1.4^{\circ}-53.9^{\circ}$ in the same three filters we discussed above.

We integrated the $I / F$ values over the comet surface using the histogram-based technique (Burger \& Burge 2013), and then divided the average $I / F$ to the visible and illuminated cross section of 67P using its shape model (Preusker et al. 2015). 


\subsection{Combined phase curve}

We combined the local and global phase curves into a single curve for corresponding filters (Fig. 4). The resulting phase curve, which we call combined phase curve, enables us to fit the photometric models to the phase curve and explore the $\mathrm{OE}$ behavior of 67P. The combined phase curve with a wide phase angle range including the data for very small phase angles is suitable for comparisons with other bodies in the solar system with an extensive range of phase angles.

The albedo map of 67P generated by Fornasier et al. (2015) and the global spectrophotometric analysis of the close-flyby images by Feller et al. (2016) justify the combination of global and local phase curves because the disk average albedo $(6.5 \pm 0.2 \%)$ and the albedo of Imhotep-Ash area $(6.15 \pm 0.07 \%)$ are close.

\section{Morphological modeling}

We used a four-parameter exponential-linear model to fit the data. The model considers the phase function as a combination of an exponential peak and a linear part. Its main interest is that it has been applied to the phase curves of various atmosphereless solar system bodies by Rosenbush et al. (2002) and references therein. The function is given by

$$
I / F=I / F_{\mathrm{s}} \exp \left(-\frac{\alpha}{1.45 \times H W H M}\right)+I / F_{\mathrm{b}}+B \alpha,
$$

where $I / F_{\mathrm{s}}$ is the amplitude of opposition peak and is defined as the brightness increase relative to the background brightness $I / F_{\mathrm{b}} . B$ is the slope of the linear part, and HWHM stands for the angular width of the OE.

Following Rosenbush et al. (2002), we also calculated the amplitude of $\mathrm{OE}$ in the form of the enhancement factor, $\zeta$. The $\zeta$ is defined as

$\zeta=\frac{I / F_{\mathrm{s}}+I / F_{\mathrm{b}}}{I / F_{\mathrm{b}}}$

We fit the model to the combined phase function of 67P for three filters (see Fig. 4) and list the best-fit values of the OE parameters, HWHM, and $\zeta$, in Table A.2.

The variation of the OE parameters with respect to the wavelength would suggest the contribution of $\mathrm{CB}$ in the formation of opposition-surge phenomenon (Mishchenko 1992). On the other hand, the independency of the OE parameters on the wavelength is typical for the $\mathrm{SH}$ effect; $\mathrm{SH}$ is a geometric optics effect; it deals with particles that are much larger than the wavelength, and it does not depend on wavelength.

The best-fit parameters obtained through modeling (see Table A.2) suggest that the wavelength dependency of the $\zeta$ and HWHM is either absent (taking into account the accuracy of the results) or that both these parameters decrease with wavelength. Neither of these behaviors are consistent with coherent backscattering, which should show a strong dependence on the wavelength, with the HWHM increasing with wavelength (Mishchenko 1992).

The albedo and color dependence of OE parameters have also been studied by Belskaya \& Shevchenko (2000). The authors stated that the contribution of CB decreases for lowalbedo asteroids, while it increases for high- and medium-albedo asteroids.

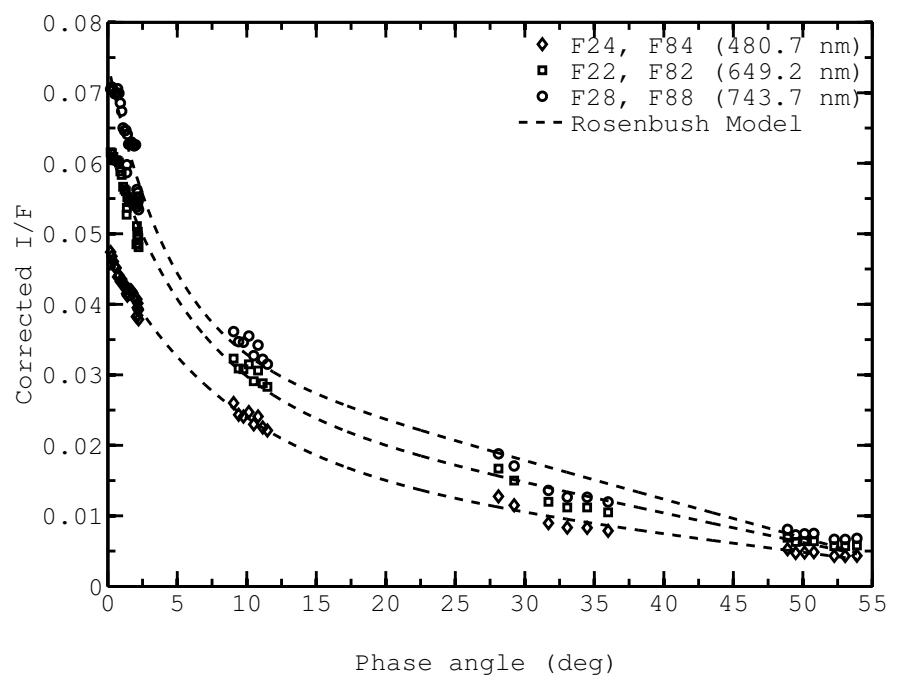

Fig. 4. Combined phase function of $67 \mathrm{P}$ in three wavelengths from OSIRIS NAC images corrected by the Lommel-Seeliger disk law. The dashed lines represent the Rosenbush et al. (2002) function stated by Eq. (3).

\section{Discussion}

We concentrate our discussion on the comparative analysis of the combined phase curves of 67P, including both disk-averaged and disk-resolved brightness. The comparison of the phase curve between bodies in the solar system and laboratory measurements enables us to constrain the physical properties of the surface of $67 \mathrm{P}$ nucleus as well as interpret the phenomenon of the opposition effect by itself.

\subsection{Comparison of 67P with bodies in the solar system}

The modeled OE parameters (HWHM and $\zeta$ ) of 67P from the combined phase curve at $\lambda=649 \mathrm{~nm}$ are plotted versus the geometric albedo (see Fig. 5) together with available OE parameters of numerous solar system bodies (Rosenbush et al. 2002). The albedo values of these bodies are also taken from Rosenbush et al. (2002) and references therein.

The resulting scatter plot (upper panel of Fig. 5) demonstrates the inverse relation of the HWHM with the geometric albedo for different airless bodies in the solar system. As the geometric albedo increases, the HWHM tends to decrease for highalbedo objects, while the HWHM data points for low-albedo object are inclined to increase although in a more scattered way. The inverse trend (dashed line) is depicted by inspection of the scatter plot of HWHM versus albedo. The modeled HWHM for 67P is located close to satellites of Mars, Phobos and Deimos, suggesting a similarity in the optical properties and structure of their surfaces, such as size of particles and porosity.

The variation of the enhancement factor, $\zeta$ against geometric albedo does not follow a recognizable trend (lower panel of Fig. 5). However, it shows two collections of data points, the values of the enhancement factor for all bright bodies studied in Rosenbush et al. (2002) are lower than 1.6, while for all dark objects they do not go beyond 1.8. It is notable that Phobos and Deimos including 67P do not resemble any group of data points.

Another comparative study can be done for 67P and existing data for Centaurs and Kuiper belts objects (KBOs) (Belskaya et al. 2008). Since these bodies are observed in the 
N. Masoumzadeh et al.: Opposition effect on comet 67P/Churyumov-Gerasimenko using Rosetta-OSIRIS images
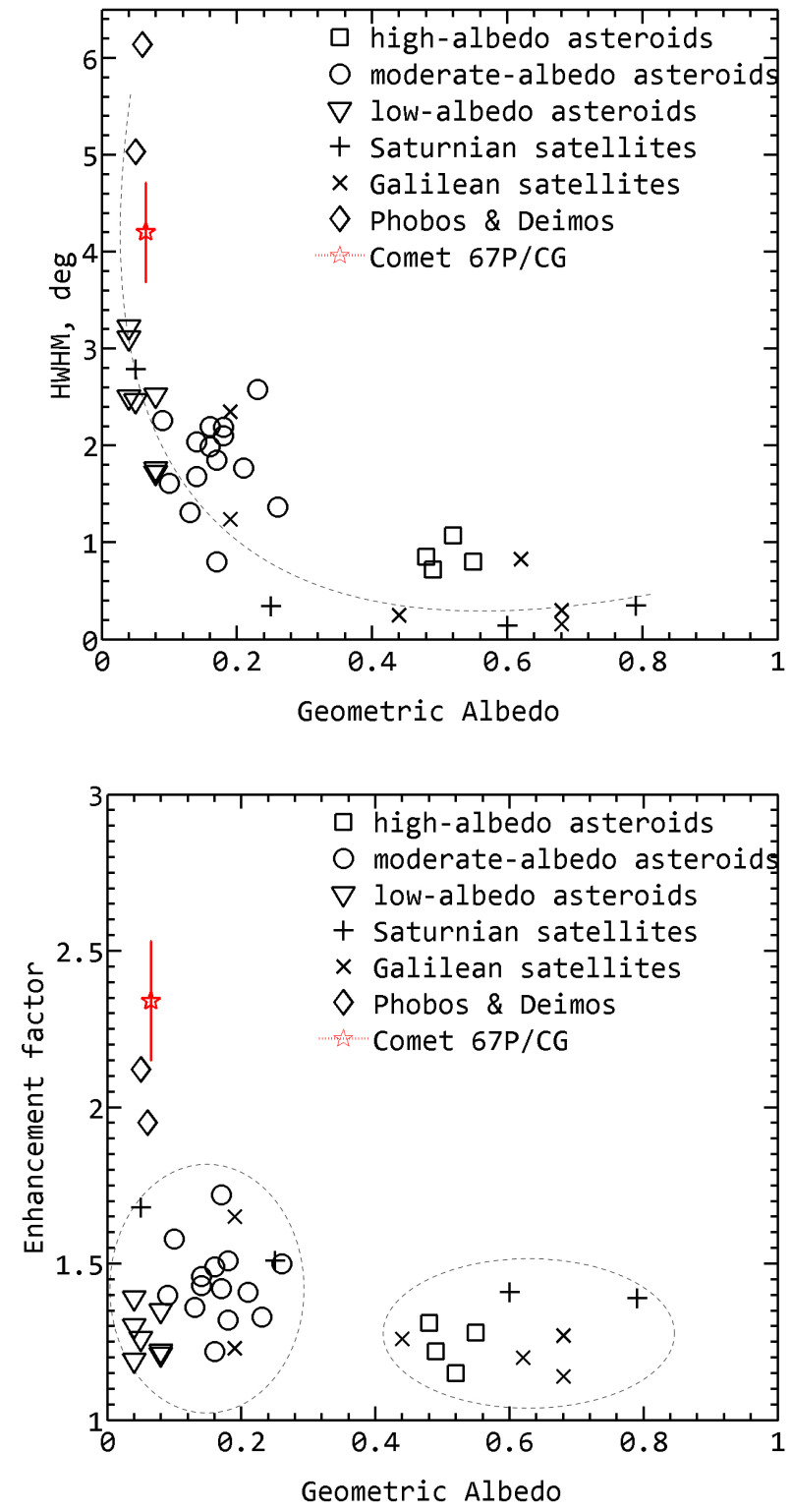

Fig. 5. Plots of the HWHM (upper panel) and the enhancement factor (lower panel) versus the geometric albedo of bodies in the solar system, evaluated by Rosenbush et al. (2002). The model OE parameters of 67P are shown by a red star symbol. The error bar is associated with the fit uncertainty. The dashed curve in the upper panel represents the inverse trend by inspection between HWHM and the geometric albedo. The dashed circular curves in the lower panel display two groups of data points with $\zeta>1.8$ (dark objects) and $<1.6$ (bright objects).

small phase angle range, for the comparison we only adopt the local phase curve of 67P at $\lambda=649 \mathrm{~nm}$ in the same phase angle range as those objects. The best-fit value of the phase coefficient in $\mathrm{mag} / \mathrm{deg}$ for $67 \mathrm{P}$ is plotted together with the measured phase coefficients of KBOs and Centaurs (Table 1 of Belskaya et al. 2008 and references therein) with respect to the albedo (see Fig. 6 ).

Figure 6 shows that $67 \mathrm{P}$ with the phase coefficient, $0.092 \pm$ 0.022 , is located in Centaurs regions. The two particular Centaurs with similar phase coefficient to 67P are (54 598) Bienor and 1999 TD10. Centaurs are believed to have a greater steepness in their phase curve than other solar system bodies (Bauer et al. 2003; Rousselot et al. 2005).

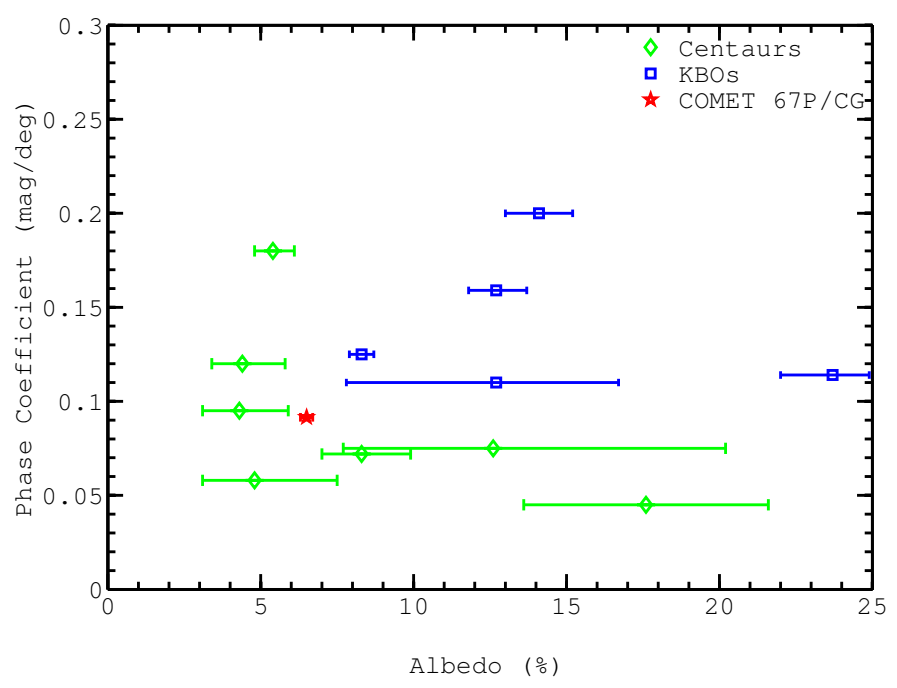

Fig. 6. Phase coefficient versus albedo for Centaurs, classical Kuiper belt objects (KBOs), and trans-Neptunian objects in $R$ band together with comet $67 \mathrm{P} / \mathrm{CG}$ at $\lambda=649 \mathrm{~nm}$.

\subsection{Comparison with laboratory measurements of the phase curve at small phase angles}

We also compared our results with laboratory measurements that study the dependence of the photometric characteristics of particulate surfaces on the surface properties, that is, albedo, grain size distribution, and porosity.

There are not many experimental studies at very small phase angles. The measurements that are made in the opposition-effect region were carried out by Jost et al. (2016) for icy particles with a high-albedo close to unity and by Psarev et al. (2007) for low- and high-albedo samples. We found that the laboratory reflectance data from carbon soot at the wavelength of $630 \mathrm{~nm}$ in the phase angle range of $0.008^{\circ}-1.5^{\circ}$ (Psarev et al. 2007) show a behavior that is very similar to the measured local phase curve from the Imhotep-Ash region of $67 \mathrm{P}$ at $\lambda=649 \mathrm{~nm}$. The local phase curve we extracted was normalized at $\alpha=1.5^{\circ}$ to make it comparable with normalized laboratory measurements (see Fig. 7).

In Fig. 7 two samples, the ultra-dispersed quartz and the carbon soot, behave similarly for the phase angles $>0.2^{\circ}$ (the limit we determined for the local phase curve of $67 \mathrm{P}$ from OSIRIS close-flyby images). However, light scattering by quartz sample is defined by its very special characteristics, such as birefringence and high albedo, which is responsible for the narrow $\mathrm{OE}$ peak at phase angles $<0.1^{\circ}$ caused by CB. Neither of them can be associated with the material in the Imhotep-Ash area, which allows us to exclude quartz from the following consideration.

The carbon soot sample has a very dark fluffy surface that contains fine grains $(\leq 1 \mu \mathrm{m})$. This resemblance as well as our previous analysis confirms that the photometric characteristics of Imhotep-Ash are typical of dark fluffy materials and are formed by the shadow-hiding effect. The results from this comparison are consistent with the conclusion of Fornasier et al. (2015) based on the global modeled Hapke parameters of 67P that the surface porosity is equal to $87 \%$. The porous regolith on $67 \mathrm{P}$ is also verified through the measurements reported by the Rosetta CONSERT experiment (Kofman et al. 2015). 


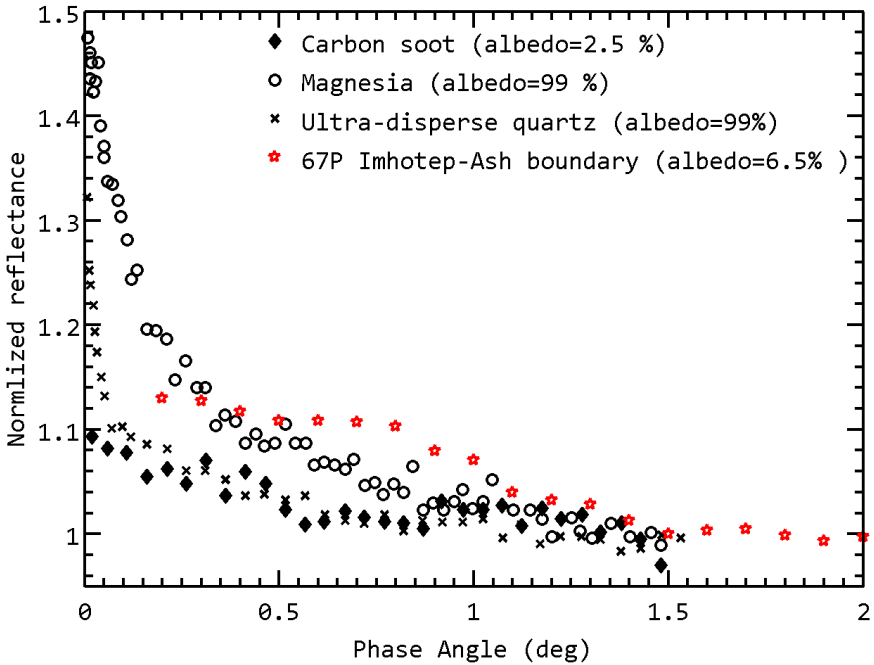

Fig. 7. Photometric phase curve for magnesia, carbon soot, and ultradispersed quartz measured in the laboratory at the $\lambda=630 \mathrm{~nm}$ in the phase angle range of $0.008^{\circ}-1.5^{\circ}$. The data points are taken from the figure in Psarev et al. (2007). The intensity is normalized at phase angle $1.5^{\circ}$. The local phase curve constructed from the Imhotep-Ash region of 67P using an OSIRIS close-flyby image in F82 $(649 \mathrm{~nm})$ filter is plotted as well (red dots).

\section{Conclusions}

This work was focused on the opposition-effect phenomenon for comet $67 \mathrm{P}$. We have extracted the surface phase function locally and globally from OSIRIS NAC images in three wavelengths. The local surface phase function was fitted by log-linear function, and the phase coefficient, $\beta$, was estimated to be $0.0915 \pm$ 0.022 at $\lambda=649.2 \mathrm{~nm}$. The resulting $\beta$ compared with Centaurs and KBOs shows that 67P belongs in the group of Centaurs.

Comparison with the existing Laboratory data at very small phase angles indicated a similar trend shown by the phase curves of the Imhotep-Ash region of $67 \mathrm{P}$ and the very dark and porous carbon soot.

We combined the local surface phase function with the global surface phase function to model the morphology of the opposition effect. The approximating function introduced by Rosenbush et al. (2002) was applied to describe the morphology of the opposition effect. The best-fit value of the morphological parameters HWHM and $\zeta$ in three filters indicates a decreasing trend with respect to wavelength, which is not expected to be caused by the coherent backscattering effect.

We collated the retrieved parameters of $67 \mathrm{P}$ with the same parameters for other bodies in the solar system explored by Rosenbush et al. (2002), which places 67P in the same category as the two satellites of Mars, Phobos and Deimos. Both Phobos and Deimos are small bodies and have spectra very similar to dark carbonaceous chondrite. Moreover, Phobos has such a low density that it may contain water ice below its surface (Fanale \& Salvail 1989, 1990). Thus, a resemblance between the opposition effect of the Martian satellites and 67P may be not a coincidence, but an indicator of some commonality in the nature of these objects.

Acknowledgements. OSIRIS was built by a consortium of the Max-PlanckInstitut für Sonnensystemforschung, Göttingen, Germany; the CISAS University of Padova, Italy; the Laboratoire d'Astrophysique de Marseille, France; the Instituto de Astrofísica de Andalucia, CSIC, Granada, Spain; the Research and Scientific Support Department of the ESA, Noordwijk, Netherlands; the Instituto Nacional de Técnica Aeroespacial, Madrid, Spain; the Universidad Politéchnica de Madrid, Spain; the Department of Physics and Astronomy of Uppsala University, Sweden; and the Institut für Datentechnik und Kommunikationsnetze der Technischen Universität Braunschweig, Germany. The support of the national funding agencies of Germany (DLR), France (CNES), Italy (ASI), Spain (MEC), Sweden (SNSB), and the ESA Technical Directorate is gratefully acknowledged. We thank the Rosetta Science Operations Centre and the Rosetta Mission Operations Centre for the successful rendezvous with comet $67 \mathrm{P} / \mathrm{Churyumov-}$ Gerasimenko. This research has made use of the USGS Integrated Software for Imagers and Spectrometers (ISIS). We gratefully acknowledge the developers of SPICE and NAIF/PDS resources. This research has made use of the webtool WwW . comet-toolbox.com

\section{References}

Acton, C. H., Jr 1996, Planet. Space Sci., 44, 65

Anderson, J. A., Sides, S. C., Soltesz, D. L., Sucharski, T. L., \& Becker, K. J. 2004, in Lunar Planet. Sci. Conf. 35, eds. S. Mackwell, \& E. Stansbery, 2039 Bauer, J. M., Meech, K. J., Fernández, Y. R., et al. 2003, Icarus, 166, 195

Belskaya, I., \& Shevchenko, V. 2000, Icarus, 147, 94

Belskaya, I., Levasseur-Regourd, A. C., Shkuratov, Y. G., \& Muinonen, K. 2008, in The Solar System Beyond Neptune, eds. M. A. Barucci, H. Boehnhardt, D. P. Cruikshank, A. Morbidelli, \& R. Dotson (Tucson: University of Arizona Press), 115

Burger, W., \& Burge, M. J. 2013, Principles of Digital Image Processing, Advanced Methods (Springer)

Ciarniello, M., Capaccioni, F., Filacchione, G., et al. 2015, A\&A, 583, A31

Déau, E. 2012, J. Quant. Spectr. Rad. Trans., 113, 1476

Déau, E., Dones, L., Rodriguez, S., Charnoz, S., \& Brahic, A. 2009, Planet. Space Sci., 57, 1282

Déau, E., Flandes, A., Spilker, L. J., \& Petazzoni, J. 2013, Icarus, 226, 1465

El-Maarry, M. R., Thomas, N., Giacomini, L., et al. 2015, A\&A, 583, A26

Fanale, F. P., \& Salvail, J. R. 1989, Geophys. Res. Lett., 16, 287

Fanale, F. P., \& Salvail, J. R. 1990, Icarus, 88, 380

Feller, C., Fornasier, S., Hasselmann, P., et al. 2016, MNRAS, 462, S287

Fornasier, S., Hasselmann, P. H., Barucci, M.A., et al. 2015, A\&A, 583, A30

Hapke, B. 1986, Icarus, 67, 264

Jorda, L., Gaskell, R., Capanna, C., et al. 2016, Icarus, 277, 257

Jost, B., Pommerol, A., Poch, O., et al. 2016, Icarus, 264, 109

Keller, H., Barbieri, C., Lamy, P., et al. 2007, Space Sci. Rev., 128, 433

Kofman, W., Herique, A., Barbin, Y., et al. 2015, Science, 349, aab0639

Lamy, P. L., Toth, I., Fernandez, Y. R., \& Weaver, H. A. 2004, in Comets II, ed. G. W. Kronk (Tucson: University of Arizona Press), 223

Li, J.-Y., Helfenstein, P., Buratti, B., Takir, D., \& Clark, B. E. 2015, in Asteroid IV, 129

Mackowski, D., \& Mishchenko, M. 2011, J. Quant. Spectr. Rad. Trans., 112, 2182

Mackowski, D. W., \& Mishchenko, M. I. 1996, J. Opt. Soc. Am. A, 13, 2266

Mishchenko, M. 1992, Astrophys. Space Sci., 194, 327

Muinonen, K., Mishchenko, M. I., Dlugach, J. M., et al. 2012, ApJ, 760, 118

Oklay, N., Vincent, J.-B., \& Fornasier, S. 2016, A\&A, 586, A80

Penttilä, A. 2013, J. Quant. Spectr. Rad. Trans., 131, 130

Preusker, F., Scholten, F., Matz, K.-D., et al. 2015, A\&A, 583, A33

Psarev, V., Ovcharenko, A., Shkuratov, Y., Belskaya, I., \& Videen, G. 2007, J. Quant. Spectr. Rad. Trans., 106, 455

Rosenbush, V., Kiselev, N., Avramchuk, V., \& Mishchenko, M. 2002, in Optics of Cosmic Dust, eds. G. Videen, \& M. Kocifaj (Springer Netherlands), NATO Science Series, 79, 191

Rousselot, P., Petit, J.-M., Poulet, F., \& Sergeev, A. 2005, Icarus, 176, 478

Schulz, R., Alexander, C., Boehnhardt, H., \& Glaßmeier, K.-H. 2009, ROSETTA, ESA's Mission to the Origin of the Solar System (New York: Springer-Verlag)

Shevchenko, V. G., Chiorny, V. G., Gaftonyuk, N. M., et al. 2008, Icarus, 196, 601

Shkuratov, Y., Ovcharenko, A., Zubko, E., et al. 2002, Icarus, 159, 396

Shkuratov, Y. G. 1994, Sol. Syst. Res., 28, 155

Thomas, N., Sierks, H., Barbieri, C., et al. 2015, Science, 347, 440

Tubiana, C., Böhnhardt, H., Agarwal, J., et al. 2011, A\&A, 527, A113

Tubiana, C., Guettler, C., Kovacs, G., et al. 2015, A\&A, 583, A46 
${ }^{1}$ Max-Planck-Institut für Sonnensystemforschung, Justus-vonLiebig-Weg 3, 37077 Göttingen, Germany

2 Centro di Ateneo di Studi ed Attivitá Spaziali "Giuseppe Colombo" (CISAS), University of Padova, via Venezia 15, 35131 Padova, Italy

3 Institute for Geophysics and Extraterrestrial Physics, TU Braunschweig, 38106 Braunschweig, Germany

4 Department of Physics and Astronomy "G. Galilei”, University of Padova, Vic. Osservatorio 3, 35122 Padova, Italy

5 Aix Marseille Université, CNRS, LAM (Laboratoire d'Astrophysique de Marseille) UMR 7326, 13388 Marseille, France

6 Centro de Astrobiologia (INTA-CSIC), European Space Agency (ESA), European Space Astronomy Centre (ESAC), PO Box 78, 28691 Villanueva de la Canada, Madrid, Spain

7 International Space Science Institute, Hallerstrasse 6, 3012 Bern, Switzerland

8 Research and Scientific Support Department, European Space Agency, 2201 Noordwijk, The Netherlands

9 Department of Physics and Astronomy, Uppsala University, Box 516, 75120 Uppsala, Sweden

10 PAS Space Research Center, Bartycka 18A, 00716 Warszawa, Poland

11 Department for Astronomy, University of Maryland, College Park, MD 20742-2421, USA

12 Gauss Professor Akademie der Wissenschaften zu Göttingen, 37077 Göttingen, Germany
13 LESIA, Observatoire de Paris, CNRS, UPMC Univ Paris 06, Univ. Paris-Diderot, 5 Place J. Janssen, 92195 Meudon Principal Cedex, France

14 LATMOS, CNRS/UVSQ/IPSL, 11 Boulevard d'Alembert, 78280 Guyancourt, France

15 INAF Osservatorio Astronomico di Padova, Vicolo dell'Osservatorio 5, 35122 Padova, Italy

16 CNR-IFN UOS Padova LUXOR, via Trasea 7, 35131 Padova, Italy

17 Department of Industrial Engineering University of Padova via Venezia, 1, 35131 Padova, Italy

18 University of Trento, via Sommarive, 9, 38123 Trento, Italy

19 Physikalisches Institut, Sidlerstrasse 5, University of Bern, 3012 Bern, Switzerland

20 INAF-Osservatorio Astronomico di Trieste, via Tiepolo 11, 34143 Trieste, Italy

21 Instituto de Astrofisica de Andalucia-CSIC, Glorieta de la Astronomia, 18008 Granada, Spain

22 German Aerospace Center (DLR), Institute of Planetary Research, 12489 Berlin-Adlershof, Germany

23 Institute for Space Science, National Central University, 32054 Chung-Li, Taiwan

24 ESA/ESAC, PO Box 78, 28691 Villanueva de la Cañada, Spain

25 Department of Information Engineering, University of Padova, via Gradenigo 6/B, 35131 Padova, Italy 


\section{Appendix A: Additional tables}

Table A.1. Characteristics of the OSIRIS NAC images used in this analysis.

\begin{tabular}{lccc}
\hline \hline Filter IDs $\left(\lambda_{\mathrm{c}}\right.$ in $\left.\mathrm{nm}\right)$ & Acquisition date & Start time $(\mathrm{UTC})$ & Phase angle $\left(^{\circ}\right)$ \\
\hline F24 (480.7), F22 (649.2), F28 (743.7) & $2014-07-25$ & $08: 12: 51.972$ & $2.2^{\circ}-2.1^{\circ}$ \\
F24, F22, F28 & $2014-07-28$ & $23: 26: 59.080$ & $1.3^{\circ}$ \\
F24, F22, F28 & $2014-07-29$ & $00: 46: 59.084$ & $1.4^{\circ}$ \\
F24, F22, F28 & $2014-08-01$ & $11: 51: 46.179$ & $9.1^{\circ}-11.5^{\circ}$ \\
F24, F22, F28 & $2014-08-02$ & $23: 22: 46.224$ & $27.0^{\circ}$ \\
F24, F22, F28 & $2014-08-03$ & $00: 22: 47.231$ & $28.1^{\circ}-35.9^{\circ}$ \\
F24, F22, F28 & $2014-08-05$ & $23: 20: 44.320$ & $48.9^{\circ}$ \\
F24, F22, F28 & $2014-08-06$ & $01: 20: 46.316$ & $50.1^{\circ}-53.9^{\circ}$ \\
F84 (480.7), F82 (649.2), F88 (743.7) & $2015-02-14$ & $12: 40: 45.838$ & $0.2^{\circ}-2.0^{\circ}$ \\
\hline
\end{tabular}

Notes. Acquisition date and time are shown for the first image of each sequence in F24 and F84.

Table A.2. Best-fit values of the OE parameters from the combined phase function of 67P based on the morphological modeling of Rosenbush et al. (2002; Eq. (3)).

\begin{tabular}{ccccc}
\hline \hline Filter IDs & $\lambda_{\mathrm{c}}(\mathrm{nm})$ & $\zeta$ & $H W H M$ & $\begin{array}{c}\text { Phase angle } \\
\text { range }(\mathrm{deg})\end{array}$ \\
\hline F24, F84 & 480.7 & $2.78 \pm 0.34$ & $5.99 \pm 0.52$ & $0.2^{\circ}-53.9^{\circ}$ \\
F22, F82 & 649.2 & $2.34 \pm 0.20$ & $4.20 \pm 0.51$ & $0.2^{\circ}-53.9^{\circ}$ \\
F28, F88 & 743.7 & $2.17 \pm 0.19$ & $3.05 \pm 0.47$ & $0.2^{\circ}-53.9^{\circ}$ \\
\hline
\end{tabular}

